\title{
The Neural Basis of Imitation is Body Part Specific
}

\author{
Georg Goldenberg ${ }^{1}$ and Hans-0tto Karnath ${ }^{2}$ \\ ${ }^{1}$ Neuropsychological Department, Bogenhausen Hospital, D-81925 Munich, Germany, and ${ }^{2}$ Section of Neuropsychology, Center of Neurology, Hertie \\ Institute for Clinical Brain Research, University of Tübingen, D-72076 Tübingen, Germany
}

Imitation is an important constituent of the behavioral repertoire of human beings. We use imitation for learning motor skills, for facilitating comprehension of other persons' actions and mental states, and as a communicative reference to actions or people. Although some non-human species display imitative behaviors, none of them equals the versatility and perfection of human imitation. The versatility and apparent ease of human imitation is, however, not at all self-evident when one considers the neural and cognitive transformations that must be accomplished for successful imitation. Imitation of meaningless gestures poses a particular challenge for our brain, because similarity between the body of the model and one's own body must bridge fundamental differences between the perspective and modalities of perceiving and controlling one's own and other bodies. We analyzed the locations of left-hemisphere lesions disturbing imitation of meaningless gestures and found a clear-cut body part specificity. Disturbed imitation of finger postures was associated with anterior lesions including the opercular portion of the inferior frontal gyrus, whereas disturbed imitation of hand postures was associated with posterior lesions affecting the inferior parietal lobule and temporo-parieto-occipital junction. These locations do not correspond with known somatotopic maps of motor representations. Rather, they argue for an intermediate step of processing interpolated between perception and execution of gestures as has been suggested by the mirror neuron and the body part coding hypotheses.

Key words: imitation; body schema; mirror neurons; inferior frontal gyrus; parietal lobe; apraxia

\section{Introduction}

Among the wide diversity of actions that human beings can imitate, meaningless gestures pose a particular challenge for our brain. Similarity between the actions of the model and one's own actions can be derived neither from their common meaning nor from the similarity of their impact on external objects. It must result from direct matching between the own body's configuration and that of the model. Such matching must bridge fundamental differences between the perspective and modality of perceiving and controlling the own and other bodies. Two basically different approaches to understanding this conversion have been proposed. One assumes direct connections from perceptual representations of other bodies to motor regions controlling the own body (Rothi et al., 1991; Buccino et al., 2001; Brass and Heyes, 2005). The other one postulates an intermediate step of neural processing. This idea has been elaborated in two major hypotheses. The hypothesis of "body part coding" proposes that a common code for perception and motor execution of gestures is produced by reference to a classification of body parts and decomposes the gestures into combinations of a limited number of defined body parts (Goldenberg, 1996; Meltzoff and Moore, 1997; Chaminade et al., 2004; Peigneux et al., 2004). The "mirror

\footnotetext{
Received Feb. 13, 2006; revised April 15, 2006; accepted April 24, 2006.

This work was supported by the Deutsche Forschungsgemeinschaft (SFB 550-A4 to H.-0.K. and 968/2-1 to G.G.) and the University of Tübingen. We are grateful to Chris Rorden and Joachim Hermsdörfer for insightful discussion and help.

Correspondence should be addressed to Dr. Georg Goldenberg, Neuropsychological Department, Bogenhausen Hospital, Englschalkingerstrasse 77, D-81925 Munich, Germany. E-mail: Georg.Goldenberg@extern.Irz-muenchen.de. D01:10.1523/JNEUROSC1.0638-06.2006

Copyright $\odot 2006$ Society for Neuroscience $\quad 0270-6474 / 06 / 266282-06 \$ 15.00 / 0$
}

neuron" hypothesis is based on the detection of neurons that fire both when a monkey observes a familiar action or when it executes the same action and postulates that these neurons support imitation of actions. In contrast to body part coding, the common code of observed and executed gestures is goal-directed actions rather than the isolated configuration of the body parts (Gallese et al., 1996; Rizzolatti and Luppino, 2001; Umilta et al., 2001; Fogassi et al., 2005).

In human beings, localized brain damage causes apraxia. Patients with apraxia commit errors when imitating gestures even when they use the nonparetic limb ipsilateral to the lesion, and this impairment affects the imitation of meaningless gestures (Kimura and Archibald, 1974; De Renzi et al., 1980; Goldenberg, 1996; Roy et al., 1996; Haaland et al., 2000; Goldenberg and Strauss, 2002). The location of lesions causing apraxia can provide clues to the cognitive and neural substrate of imitation. It has been shown that the laterality of crucial lesions is body part specific. Dissociations have been documented between postures of limbs and face, upper and lower face, and fingers, hand, and foot (Raade et al., 1991;Bizzozero et al., 2000; Goldenberg and Strauss, 2002). Whereas left brain damage can affect imitation for all of these body parts, right brain damage interferes only with postures of the upper face, the feet, and the fingers (Goldenberg, 1996; Bizzozero et al., 2000; Goldenberg and Strauss, 2002; Lausberg and Cruz, 2004). Nevertheless, the exact intrahemispheric locations of critical cortical areas are not yet known. The present study aimed to identify the neural structures supporting imitation of hand and finger postures. Because only left brain damage affects both types of gestures, we restricted the present study to these patients. 
Table 1. Demographic and clinical data of patients

\begin{tabular}{|c|c|c|c|c|c|c|c|c|c|c|c|c|}
\hline & Gender & Age & $\begin{array}{l}\text { Time since } \\
\text { lesion } \\
\text { (weeks) }\end{array}$ & $\begin{array}{l}\text { Hand } \\
\text { imitation }\end{array}$ & $\begin{array}{l}\text { Finger } \\
\text { imitation }\end{array}$ & Aphasia & $\begin{array}{l}\text { Token } \\
\text { test }^{a}\end{array}$ & Repetition $^{a}$ & $\begin{array}{l}\text { Written } \\
\text { language }^{a}\end{array}$ & Naming $^{a}$ & Comprehension $^{a}$ & $\begin{array}{l}\text { Lesion } \\
\text { size }^{b}\end{array}$ \\
\hline All patients ${ }^{c}$ & $17 \mathrm{~F} / 27$ & $51.2(13.3)$ & $19.3(22.9)$ & 14.8 (4.6) & $14.7(4.3)$ & $\begin{array}{l}19 \text { global, } 4 \text { Broca, } \\
6 \text { Wernicke, } \\
9 \text { amnesic, } \\
6 \text { other }\end{array}$ & $44.1(9.4)$ & $43.6(8.5)$ & 44.7 (7.5) & $43.3(7.9)$ & $46.8(8.8)$ & $15.9(8.2)$ \\
\hline \multirow{7}{*}{$\begin{array}{c}\text { Hand defective } \\
\text { but finger } \\
\text { normal }\end{array}$} & M & 68 & 4 & 1 & 20 & Wernicke & 40 & 37 & 39 & 41 & 32 & 15.3 \\
\hline & M & 53 & 16 & 10 & 17 & Global & 49 & 49 & 44 & 44 & 46 & 35.0 \\
\hline & $\mathrm{F}$ & 55 & 12 & 11 & 19 & Amnesic & 49 & 50 & 39 & 48 & 40 & 10.0 \\
\hline & M & 48 & 10 & 14 & 17 & Wernicke & 42 & 42 & 50 & 43 & 41 & 5.5 \\
\hline & M & 68 & 26 & 15 & 18 & Global & 49 & 49 & 42 & 34 & 42 & 13.4 \\
\hline & $\mathrm{F}$ & 26 & 8 & 16 & 20 & Broca & 53 & 42 & 47 & 49 & 52 & 13.0 \\
\hline & $\mathrm{F}$ & 31 & 11 & 16 & 20 & Amnesic & 39 & 41 & 54 & 63 & 57 & 14.6 \\
\hline \multirow{5}{*}{$\begin{array}{l}\text { Finger } \\
\text { defective } \\
\text { but hand } \\
\text { normal }\end{array}$} & $F$ & 66 & 6 & 18 & 9 & Global & 44 & 44 & 34 & 34 & 45 & 21.9 \\
\hline & M & 41 & 26 & 19 & 12 & Global & 29 & 29 & 43 & 39 & 42 & 7.8 \\
\hline & M & 58 & 19 & 19 & 13 & Wernicke & 29 & 29 & 34 & 34 & 34 & 17.8 \\
\hline & M & 65 & 16 & 19 & 15 & Amnesic & 40 & 40 & 41 & 38 & 42 & 23.4 \\
\hline & M & 48 & 20 & 20 & 13 & Broca & 49 & 50 & 49 & 56 & 60 & 29.7 \\
\hline
\end{tabular}

F, Female; $M$, male.

aSubtests of the Aachen Aphasia Test: $t$ values.

${ }^{b}$ Percentage of left-hemisphere volume.

'Data are mean (SD). A selective deficit of imitating either hand or finger postures was diagnosed when the scores were below cutoff for one of them and above for the other, and when the numerical difference between them was $>2$.
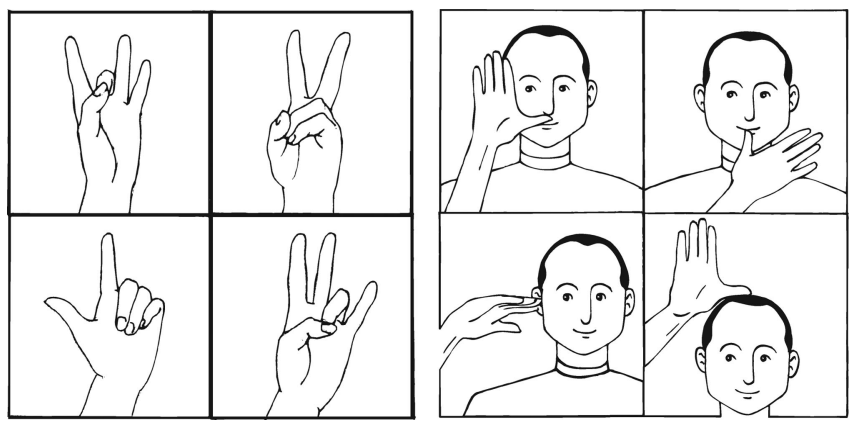

Figure 1. Examples of the hand and finger postures used for assessment of imitation.

\section{Materials and Methods}

Patients. Forty-four patients admitted to the Neuropsychological Department of Bogenhausen Hospital were examined. Consecutive righthanded patients who had suffered a left-sided stroke at least 3 weeks before and who had no magnetic resonance imaging (MRI) evidence of diffuse or bilateral lesions were included. All patients had a complete Aachen Aphasia Test (Huber et al., 1983) within 14 d from the experimental tests. All patients or their relatives gave their informed consent for participation in the study, which was performed in accordance with the ethical standards of the 1964 Declaration of Helsinki. Demographic and clinical data are displayed in Table 1.

Imitation of hand and finger postures. Imitation was tested for two kinds of meaningless gestures (Fig. 1): 10 hand postures required the patients to copy different positions of the hand relative to the head and face while the configuration of the fingers remained invariant. For imitation of 10 finger postures, patients were asked to replicate different configurations of the fingers, whereas the position of the entire hand relative to the body was not considered for scoring.

To exclude the influence of motor and somatosensory deficits on the accuracy of imitation, patients used their ipsilesional left arm and hand, while the examiner demonstrated the gestures "like a mirror" with his right hand. Correct imitation on the first trial yielded two points. Otherwise, the demonstration was repeated and 1 point was given for a successful second trial. Scoring considered only the final position of the relevant body part and did not take into account hesitation, searching movements, or self-corrections during the course of the movement. In a previous study using the same test, inter-rater agreement of scoring was
92\% for hand and 99\% for finger postures (Goldenberg and Strauss, 2002). For determination of cutoff scores, we used previously published data obtained by administration of the same test to 60 healthy controls (23 females and 37 males; mean age, 54.4 years; SD, 14.0) (Goldenberg, 1996). Imitation was classified as disturbed when below the fifth percentile of the control group ( 18 of 20 for hand, 17 of 20 for fingers).

Lesion analysis. MRI scans were obtained at the radiological service of Bogenhausen Hospital on a 1.0 T MR system (Magnetom Expert; Siemens, Erlangen, Germany). The T2 sequence used for analysis of lesion location was acquired with 19 axial slices (thickness, $5 \mathrm{~mm}$; interslice gap, $1.5 \mathrm{~mm}$ ), a field of view of $201 \times 230 \mathrm{~mm}^{2}$, a matrix size of $224 \times 512$, a repetition time of $3600 \mathrm{~ms}$, and an echo time of $96 \mathrm{~ms}$. The minimum time between stroke and imaging was 3 weeks; the average period for the 44 patients was 16.4 weeks (SD, 17.0).

Mapping of lesions was performed by one experimenter (H.-O.K.) without knowledge of test results and clinical features of the patients. Lesions were mapped using MRIcro software (Rorden and Brett, 2000) (http://www.sph.s.c..edu/comd/rorden/mricro.html) on slices of a T1weighted template MRI scan from the Montreal Neurological Institute (www.bic.mni.mcgill.ca/cgi/icbm_view). This template is approximately oriented to match Talairach space (Talairach and Tournoux, 1988) and is distributed with MRIcro. The template scan provides various anatomical landmarks for precisely plotting size and localization of the lesion. Lesions were mapped onto the slices that correspond to $z$ coordinates -40 , $-32,-24,-16,-8,0,8,16,24,32,40$, and $50 \mathrm{~mm}$ in Talairach coordinates by using the identical or the closest matching transversal slices of each individual.

To identify regions relevant for a dysfunction, we used lesion subtraction analysis (cf. Rorden and Karnath, 2004). Anatomical studies that simply superimpose lesions from patients who show the disorder of interest (disturbed imitation in the present case) may reflect vulnerability of certain regions to injury (e.g., because of the vasculature of these regions) rather than any direct involvement with this disorder. Thus, it is necessary to contrast directly the lesion sites of these patients with those of control patients who do not exhibit the disorder of interest but show a lesion in the same hemisphere and are comparable with respect to other relevant neurological and neuropsychological variables. Lesion subtraction plots directly contrast stroke patients showing the disorder of interest (a lesion overlay with positive values) with a control group (a lesion overlay with negative values). The resulting subtraction image highlights regions that are both frequently damaged in the patients showing the disorder of interest as well as being typically spared in control patients. 
The relative incidence of damage to regions unrelated to the disorder of interest should be equally represented in both patient groups and will therefore not be highlighted in subtraction plots. Subtraction analysis thus is an essential tool for exploring the critical brain structures associated with a cognitive function (for details, see Rorden and Karnath, 2004).

Because subtractions were made between groups of different sizes, we used proportional values. Automatic three-dimensional rendering of the lesion data were performed using MRIcro.

\section{Results}

In a first step of analysis, we considered only those patients from our entire sample who had a selective impairment of either hand or finger postures. There were seven patients in whom defective imitation of hand postures contrasted with normal imitation of finger postures and five patients with the reverse dissociation. Table 1 shows demographic and clinical data of these patients, and Figure $2 a$ shows the simple overlay of their lesion locations. They did not significantly differ with respect to age, gender distribution, time since lesion, lesion size, distribution of types of aphasia, and severity of impairment on the subtests of the Aachen Aphasia Test (Table 1).

To identify the structures that were specifically damaged in patients with a selective disturbance of imitation of hand postures and in patients with a selective disturbance of imitation of finger postures, we subtracted the superimposed lesions of the latter group from the overlap image of the former. The subtraction image (Fig. $2 b$ ) shows regions that were commonly damaged in patients with disturbed hand imitation but spared in patients with disturbed finger imitation in warm (red to yellow) colors and regions that were damaged in the patients with disturbed finger imitation but spared in those with disturbed hand imitation in cold (blue) colors.

A clear anatomical double dissociation corresponded to the behavioral double dissociation. The lesion overlap of patients with selectively disturbed imitation of hand postures were centered on the middle temporal and middle occipital gyri as well as on the inferior parietal lobule (IPL). It extended into the underlying temporoparietal white matter. In contrast, the center of lesion overlap for patients with selectively disturbed imitation of finger postures was located in the inferior frontal gyrus (IFG) and the adjacent insular cortex with subcortical extension into the putamen and caudate nucleus.

Lesions causing selective impairment of imitation of either
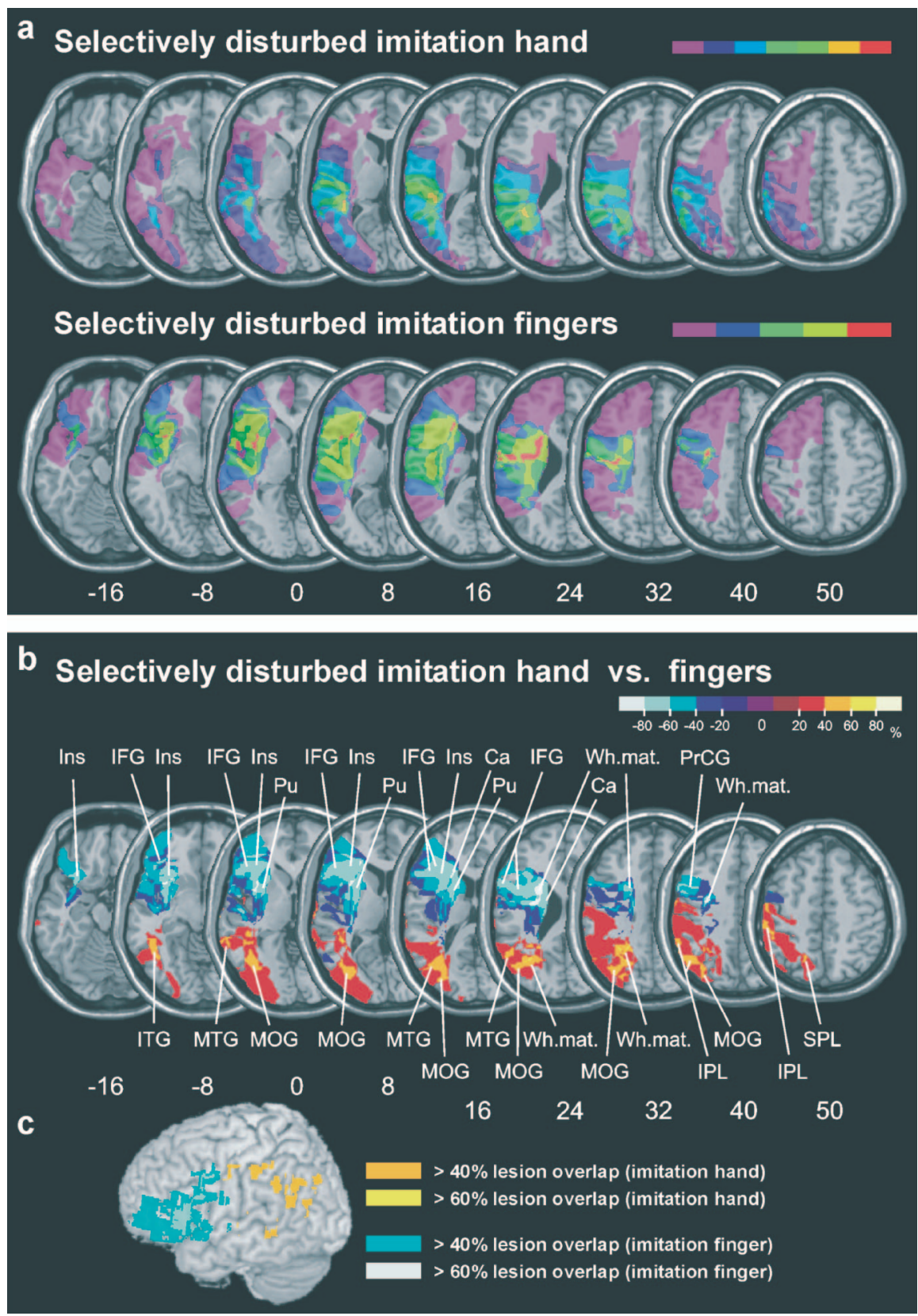

Figure 2. $\quad \boldsymbol{a}, 0$ verlay lesion plots of the patients with selective disturbances of only imitation of hand postures $(n=7)$ or of only imitation of finger postures $(n=5)$. The number of overlapping lesions is illustrated by different colors coding increasing frequencies from violet $(n=1)$ to red ( $n$ is the maximum number of subjects in the respective group). Talairach $z$ coordinates 29 of the transverse sections are given. $\boldsymbol{b}$, Plot of the subtracted superimposed lesions of the group of patients with selective disturbance of hand imitation $(n=7)$ minus the group with selectively disturbed finger imitation $(n=5)$. The percentage of overlapping lesions of the patients with disturbed hand imitation after subtraction of the group with disturbed finger imitation is illustrated by five different colors coding increasing frequencies from dark red (difference, 1-20\%) to bright yellow (difference, $81-100 \%$ ). Each color represents $20 \%$ increments. The different colors from dark blue (difference, -1 to $-20 \%$ ) to light blue (difference, -81 to $-100 \%$ ) indicate regions damaged more frequently in the group of patients with selective disturbance of finger imitation than in the group with selective disturbance of imitation of hand postures. Talairach coordinates (Talairach and Tournoux, 1988) of the transverse sections are given. Ins, Insula; Pu, putamen; Ca, caudate nucleus; PrCG, precentral gyrus; ITG, inferior temporal gyrus; MTG, middle temporal gyrus; MOG, middle occipital gyrus; SPL, superior parietal lobule; Wh.mat., white matter. c, Lateral surface view of the centers of lesion overlap in the patient group with disturbed imitation of finger postures and in the group with disturbed imitation of hand postures.

finger or hand postures need not necessarily reveal the entire extension of the regions necessary for each of them. There might be additional structures in which lesions cause impairment of both types of imitation. The selection of patients who were impaired in only one of them could exclude patients whose impair- 

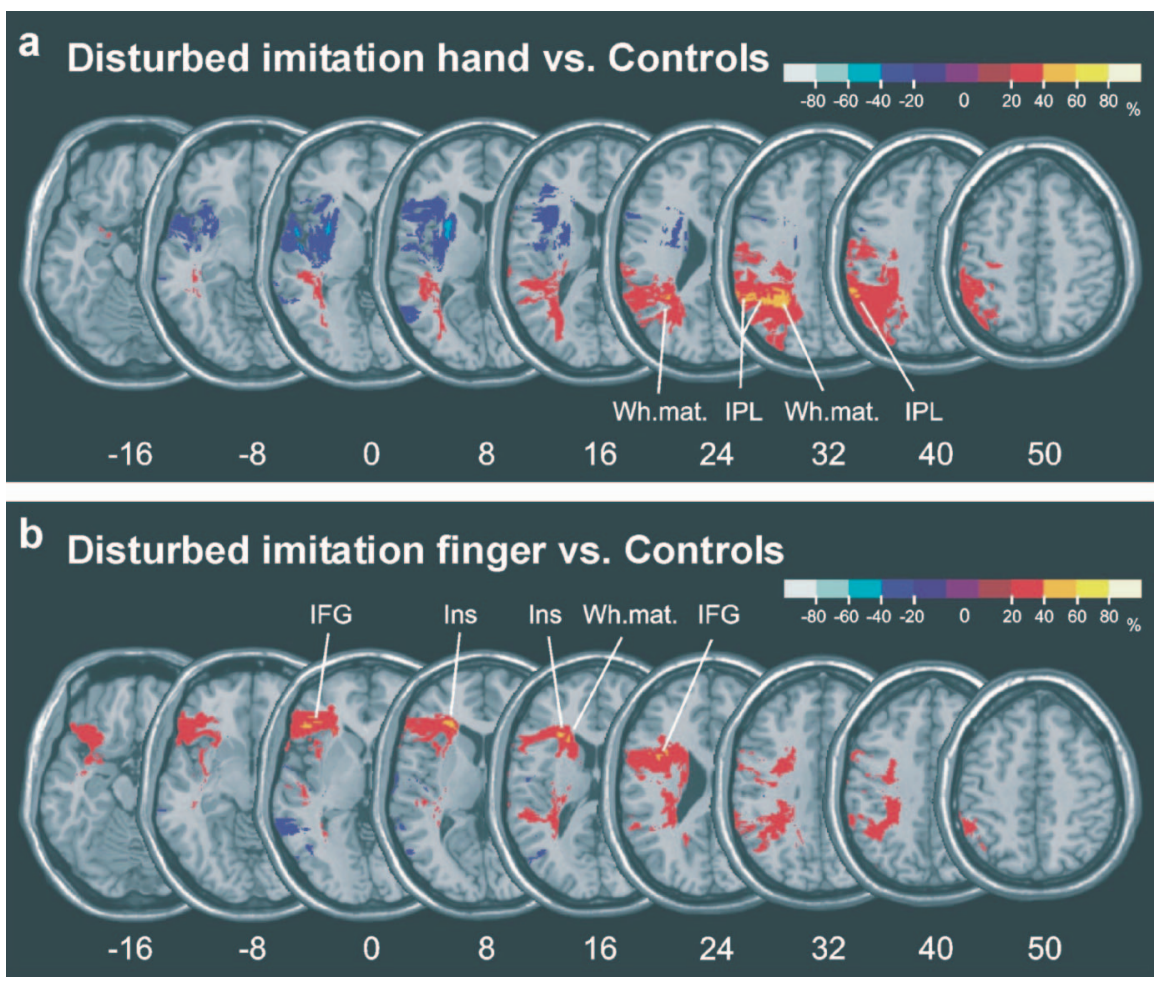

Figure 3. $\quad \boldsymbol{a}$, Plots of the subtracted superimposed lesions of the patients with disturbed imitation of hand postures $(n=28)$ minus the respective control group $(n=16)$ who were unimpaired. $\boldsymbol{b}$, Plots of the subtracted superimposed lesions of the patients with disturbed imitation of finger postures $(n=25)$ minus the respective control group $(n=19)$ who were unimpaired. The percentages of overlapping lesions in the groups with defective imitation after subtraction of their respective control group are illustrated by five different colors coding increasing frequencies from dark red (difference, 1-20\%) to bright yellow (difference, $81-100 \%)$. Each color represents $20 \%$ increments. The different colors from dark blue (difference, -1 to $-20 \%$ ) to light blue (difference, -81 to $-100 \%$ ) indicate regions damaged more frequently in the control groups than in the groups with defective imitation. Talairach coordinates (Talairach and Tournoux, 1988) of the transverse sections are given. Ins, Insula; Wh.mat., white matter.

ment on both tests is caused by damage to these common territories and thus veil its importance for mastering the task. Therefore, we conducted two additional analyses with the entire sample of 44 patients, in which we subtracted for each of the two functions the lesions of patients who performed it normally from those with disturbance.

Imitation of hand postures was defective in 28 patients and normal in 16 patients. Subtraction of the lesion overlay of unimpaired patients from that of the group with impairment revealed a center of lesion overlap associated with impaired imitation of hand postures in the IPL and the underlying white matter (Fig. $3 a$ ). Imitation of finger postures was defective in 25 and normal in 19 patients. Subtraction of lesion overlay of unimpaired patients from those with impairment revealed the center of overlap associated with impaired imitation of finger postures in the IFG and adjacent insular cortex (Fig. $3 b$ ). Both analyses thus yielded centers of overlap within the territories found by comparison between patients with selective impairment of only hand and only finger postures.

\section{Discussion}

On a behavioral level, our results complement previous demonstrations of a double dissociation of defective imitation of hand and finger postures between patients with left and right brain damage (Goldenberg, 1996; Goldenberg and Strauss, 2002) by demonstrating that such double dissociation exists also between patients with left brain damage. It was associated with a double dissociation of responsible lesions within the left hemisphere. Disturbed imitation of finger postures was contingent on anterior lesions including the IFG and insula with subcortical extension into the putamen and caudate nucleus, whereas disturbed imitation of hand postures was associated with posterior lesions affecting the junction of middle temporal and middle occipital gyri with the IPL [i.e., the temporo-parieto-occipital (TPO) junction] and extending into the underlying temporo-parietal white matter. We thus conclude that the double dissociation between lesions causing impaired imitation of hand and of finger postures indicates distinct neural substrates for these apparently very similar imitation tasks.

Our finding is corroborated by functional imaging studies demonstrating activation of IFG by imitation. Without exception, these studies have probed imitation of finger movements (Decety et al., 1997; Iacoboni et al., 1999; Koski et al., 2003, 2002; Tanaka and Inui, 2002; Heiser et al., 2003; Makuuchi, 2005). Tanaka and Inui (2002) directly compared imitation of finger and hand postures and found activation of IFG exclusively for imitation of finger postures. Haaland et al. (2000) applied analysis of lesion overlap to explore the neural substrate of imitation deficits. Their gestures combined finger and hand postures, but they distinguished between "target errors" of hand position and "internal hand position" errors of finger postures. None of four patients with exclu-

sively anterior lesions but four of five patients with exclusively posterior lesions committed target errors, whereas internal errors were present in all of the patients with anterior lesions but only three of the five patients with posterior lesions.

The locations representing imitation of finger and of hand postures are not easily accounted for by direct links from perceptual to motor representations of actions. On the perceptual side, neurons in the superior temporal cortex (STS) are tuned to various combinations of identity, configuration, and movement of body parts (Carey et al., 1997; Downing et al., 2001; Beauchamp et al., 2003; Puce and Perrett, 2003; Haasson et al., 2004). It might well be that different populations of cells within the STS react to hand and finger configurations, but because we found no influence of STS lesions on imitation at all, such perceptual specialization is unlikely to account for the body part specificity of defective imitation. Neurons in the premotor and motor cortex are typically arranged in continuous somatotopic maps. Body partspecific impairment of imitation could be accounted for by partial destruction of the somatotopic motor map, which causes defective imitation for only the body parts represented in the affected portion. Our results argue against this possibility because the arrangement of lesions responsible for disturbed imitation of finger and hand postures did not correspond to upper limb representations in known somatotopic motor maps (Creutzfeld, 1983; Buccino et al., 2001; Picard and Strick, 2001; Rizzolatti and 
Luppino, 2001). Moreover, the distance between areas responsible for finger and for hand postures is hardly compatible with a somatotopic representation of these adjacent body parts.

The opercular part of the IFG where we found the center of lesion overlap for disturbed imitation of finger postures has been proposed to be the homolog of monkey area F5, where mirror neurons were first described in the monkey. They react to goaldirected actions of the hand such as grasping or manipulating food. Selection of the appropriate hand shape is an essential feature of such actions. Because the repertoire of finger configurations and resulting hand shapes is larger in man than in nonhuman primates, human mirror neurons presumably can distinguish many more hand shapes than those of monkey. Imitation of finger postures could be achieved by matching the demonstrated posture to a similar hand shape within the repertoire of hand-object interactions stored in mirror neurons. The mirror neurons could then coordinate the motor programs for formation of the same hand shape (Rizzolatti and Luppino, 2001).

The location of lesions responsible for defective imitation of hand postures at the TPO is more difficult to reconcile with a prominent role of mirror neurons, although recent studies have suggested the existence of such neurons in the parietal lobes. They are supposed to code the goal of actions rather than the specific hand-object interactions used for attaining it and forward this information to IFG neurons coding the details of the hand-object interaction (Fogassi and Luppino, 2005; Fogassi et al., 2005; Iacoboni et al., 2005). This mechanism implies that parietal mirror neurons cannot perform their function without a connection to intact IFG neurons and, consequently, that destruction of IFG neurons cannot be compensated by intact parietal ones. It thus cannot explain the apparent independence of hand posture imitation from integrity of the IFG, and it does not readily yield an explanation why integrity of parietal regions is necessary for hand but not for finger postures.

Like the hypothesis that imitation is based on mirror neurons, the hypothesis of body part coding postulates the existence of a common code linking perception and execution of gestures, but this common code describes the configuration of the body rather than its interaction with external objects. Based on knowledge about their distinctive features and boundaries, the body parts involved in the gestures are categorized and the gestures are coded as simple spatial relationships between a limited set of discrete body parts (Goldenberg, 1996; Meltzoff and Moore, 1997; Goldenberg et al., 2001; Goldenberg and Strauss, 2002; Chaminade et al., 2004; Peigneux et al., 2004). Such body part coding produces equivalence between demonstration and imitation that is independent of the different modalities and perspectives of perceiving one's own and other persons' bodies, and it reduces the load on working memory in which the shape of the gesture must be held until motor execution is completed.

Body part coding poses different kinds of difficulty for imitation of hand and of finger postures. Positions of the hand relative to parts of the head are combinations of several hand orientations with a multitude of body parts such as the chin, lips, back, or tip of the nose, cheek, or ears. The body parts involved differ from each other by many visual features. In contrast, finger configurations are composed of a very limited set of uniform elements that differ only in their serial position. The resulting similarity between different finger configurations makes them vulnerable to interference and renders selection of the currently correct one difficult. The main difficulty of hand postures thus resides in the great amount of visual information that has to be classified and maintained from perception to motor replication of gestures, whereas the main difficulty of finger postures concerns selection from a very restricted range of very similar configurations.

This task analysis locates the crucial difficulty of imitation at an intermediate step between perception and reproduction of gestures, in which the perceived gesture is recoded and maintained in working memory until motor execution is completed. It is compatible with known functions of the regions, the integrity of which seems to be necessary for correct imitation. An increase in activity in the inferior frontal lobe has been documented in relation to increased demands on selection among competing contents of working memory (Rowe et al., 2000; ThompsonSchill, 2003; Zhang et al., 2004). Lesions of the posterior portion of the left IPL are a regular finding in patients suffering from autotopagnosia (i.e., the inability to locate body parts either on their own or on other person's bodies). Errors occur independently of whether body parts are designated verbally or by visual demonstration. Apparently, these patients cannot access the knowledge about the classification and location of body parts that, according to the body part coding hypothesis, would also be needed for decomposing gestures into simple relationships between a limited number of defined body parts (Goldenberg, 2002). Interestingly, identification of single fingers can be preserved in patients with autotopagnosia (De Renzi and Scotti, 1970; Poncet et al., 1971; Assal and Butters, 1973).

Our study considered only a small subset from the possible range of gestures and body configurations that can be imitated. It is very likely that postures of other parts of the body or sequences of postures engage brain regions not involved by imitation of simple hand and finger postures. Nevertheless, we found a double dissociation even between these two very similar kinds of gestures. A main conclusion of our study thus is that the neural substrate of imitation varies significantly with the nature of the gesture that is being imitated.

\section{References}

Assal G, Butters J (1973) Troubles du schéma corporel lors des atteintes hémisphériques gauches. Schweizer Medizinische Rundschau 62:172-179.

Beauchamp MS, Lee KE, Haxby JV, Martin A (2003) Parallel visual motion processing streams for manipulable objects and human movements. Neuron 34:149-159.

Bizzozero I, Costato D, Della Sala S, Papagno C, Spinnler H, Venneri A (2000) Upper and lower face apraxia: role of the right hemisphere. Brain 123:2213-2230.

Brass M, Heyes C (2005) Imitation: is cognitive neuroscience solving the correspondence problem? Trends Cogn Sci 9:489-495.

Buccino G, Binkowski F, Fink GR, Fadiga L, Fogassi L, Gallese V, Seitz RJ, Zilles K, Rizzolatti G, Freund HJ (2001) Action observation activates premotor and parietal areas in a somatotopic manner: an fMRI study. Eur J Neurocsi 13:400-404.

Carey DP, Perrett DI, Oram MW (1997) Recognizing, understanding and reproducing action. In: Handbook of neuropsychology, Vol 11 (Boller F, Grafman J, eds), pp 111-129. Amsterdam: Elsevier Science.

Chaminade T, Meltzoff AN, Decety J (2004) An fMRI study of imitation: action representation and body schema. Neuropsychologia 43:115-127.

Creutzfeld OD (1983) Cortex Cerebri-Leistung, strukturelle und funktionelle Organisation der Hirnrinde. Berlin: Springer.

Decety J, Grèzes J, Costes N, Perani D, Jeannerod M, Procyk E, Grassi F, Fazio F (1997) Brain activity during activation of actions - influence of action content and subject's strategy. Brain 120:1763-1777.

De Renzi E, Scotti G (1970) Autotopagnosia: fiction or reality? Arch Neurol 23:221-227.

De Renzi E, Motti F, Nichelli P (1980) Imitating gestures-A quantitative approach to ideomotor apraxia. Arch Neurol 37:6-10.

Downing PE, Jiang Y, Shuman M, Kanwisher N (2001) A cortical area selective for visual processing of the human body. Science 293:2470-2473. 
Fogassi L, Luppino G (2005) Motor functions of the parietal lobe. Curr Opin Neurobiol 15:626-631.

Fogassi L, Ferrari PF, Gesierich B, Rozzi S, Chersi F, Rizzolatti G (2005) Parietal lobe: from action organization to intention understanding. Science 308:662-667.

Gallese V, Fadiga L, Fogassi L, Rizzolatti G (1996) Action recognition in the premotor cortex. Brain 119:593-609.

Goldenberg G (1996) Defective imitation of gestures in patients with damage in the left or right hemisphere. J Neurol Neurosurg Psychiatry 61:176-180.

Goldenberg G (2002) Body perception disorders. In: Encyclopedia of the human brain, Vol 1 (Ramachandran VS, ed), pp 443-458. San Diego: Academic.

Goldenberg G, Strauss S (2002) Hemisphere asymmetries for imitation of novel gestures. Neurology 59:893-897.

Goldenberg G, Hermsdörfer J, Laimgruber K (2001) Imitation of gestures by disconnected hemispheres. Neuropsychologia 39:1432-1443.

Haaland KY, Harrington DL, Knight RT (2000) Neural representations of skilled movement. Brain 123:2306-2313.

Haasson U, Nir Y, Levy I, Fuhrmann G, Malach R (2004) Intersubject synchronization of cortical activity during natural vision. Science 303:1634-1640.

Heiser M, Iacoboni M, Maeda F, Marcus J, Mazziotta JC (2003) The essential role of Broca's area in imitation. Eur J Neurocsi 17:1123-1128.

Huber W, Poeck K, Weniger D, Willmes K (1983) Aachener Aphasie Test. Goettingen: Hogreve.

Iacoboni M, Woods RP, Brass M, Bekkering H, Mazziotta JC, Rizzolatti G (1999) Cortical mechanisms of human imitation. Science 286:2526-2528.

Iacoboni M, Molnar-Szakacs I, Gallese V, Buccino G, Mazziotta JC, Rizzolatti G (2005) Grasping the intentions of others with one's own mirror neuron system. PLoS Biol 3:e79.

Kimura D, Archibald Y (1974) Motor functions of the left hemisphere. Brain 97:337-350.

Koski L, Iacoboni M, Dubeau MC, Woods RP, Mazziotta JC (2003) Modulation of cortical activity during different imitative behaviors. J Neurophysiol 89:460-471.

Koski L, Wohlschläger A, Bekkering H, Woods RP, Dubeau MC, Mazziotta JC, Iacoboni M (2002) Modulation of motor and premotor activity during imitation of target directed action. Cereb Cortex 12:847-855.

Lausberg H, Cruz RF (2004) Hemispheric specialisation for imitation of hand-head positions and finger configurations: a controlled study in patients with complete callosotomy. Neuropsychologia 42:320-334.
Makuuchi M (2005) Is Broca's area crucial for imitation? Cereb Cortex 15:563-570.

Meltzoff AN, Moore MK (1997) Explaining facial imitation: a theoretical model. Early Dev Parent 6:179-192.

Peigneux P, Van der Linden M, Garraux G, Laureys S, Degueldre C, Aerts J, Del Fiore G, Moonen G, Luxen A, Salmon E (2004) Imaging a cognitive model of apraxia: the neural substrate of gesture-specific cognitive processes. Hum Brain Mapp 21:119-142.

Picard N, Strick PL (2001) Imaging the premotor areas. Curr Opin Neurobiol 11:663-672.

Poncet M, Pellissier JF, Sebahoun M, Nasser CJ (1971) A propos d'un cas d'autotopagnosie secondaire à une lésion pariéto-occipitale de l'hémisphère majeur. Encéphale 61:1-14.

Puce A, Perrett DI (2003) Electrophysiology and brain imaging of biological motion. Philos Trans R Soc Lond B Biol Sci 358:435-445.

Raade AS, Rothi LJG, Heilman KM (1991) The relationship between buccofacial and limb apraxia. Brain Cogn 16:130-146.

Rizzolatti G, Luppino G (2001) The cortical motor system. Neuron 31:889-901.

Rorden C, Brett M (2000) Stereotaxic display of brain lesions. Behav Neurol 12:191-200.

Rorden C, Karnath HO (2004) Using human brain lesions to infer function: a relic from a past era in the fMRI age? Nat Rev Neurosci 5:813-819.

Rothi LJG, Ochipa C, Heilman KM (1991) A cognitive neuropsychological model of limb praxis. Cogn Neuropsychol 8:443-458.

Rowe JB, Toni I, Josephs O, Frackowiak RSJ, Passingham RE (2000) The prefrontal cortex: response selection or maintenance within working memory? Science 288:1656-1660.

Roy EA, Black SE, Winchester TR, Barbur JL (1996) Gestural imitation following stroke. Brain Cogn 30:343-346.

Talairach J, Tournoux P (1988) Co-planar stereotaxic atlas of the human brain: 3-dimensional proportional system—an approach to cerebral imaging. New York: Thieme.

Tanaka S, Inui T (2002) Cortical involvement for action imitation of hand/ arm postures versus finger configurations: an fMRI study. NeuroReport 13:1599-1602.

Thompson-Schill SL (2003) Neuroimaging studies of semantic memory: inferring "how" from "where." Neuropsychologia 41:280-292.

Umilta MA, Kohler E, Gallese V, Fogassi L, Fadiga L, Keysers C, Rizzolatti G (2001) I know what you are doing: a neurophysiological study. Neuron 31:155-165.

Zhang JX, Feng CM, Fox PT, Gao JH, Tan LH (2004) Is left inferior frontal gyrus a general mechanism for selection? NeuroImage 23:596-603. 